\title{
Virtual Instruments for Urban Design Decision-Making Process
}

\author{
Marc Aurel Schnabel* and Shuva Chowdhury \\ Faculty of Architecture \& Design, Victoria University of Wellington, New Zealand \\ *Corresponding author: Marc Aurel Schnabel, Faculty of Architecture \& Design, Victoria University of Wellington, New Zealand
}

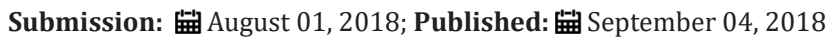

\begin{abstract}
The paper presents a framework to develop participatory urban design decision-making interfaces for the early stage of design. We produce a parametric integrated system to generate urban configurations that relate to functional areas, land-divisions, construction cost and possible revenue. We speculate this kind of interface can engage stakeholders in urban design decision-making processes. The paper presents instruments to cater urban parameters according to the interests of the designer and need of the design context. As a proof of concept, we have tested the interface in a low-density suburb of Wellington, New Zealand. We conclude the paper with an overview of our virtual design communication instruments that offer further participatory engagement throughout an urban design process.
\end{abstract}

Keywords: Parametric urban design; Decision-making; Virtual reality; Urban form

\section{Introduction}

Our study develops a method to create user-friendly interfaces to generate and visualise urban form. Typically, conventional urban design processes do not have instruments that can visualise urban form in real time during the decision-making stage. Virtual environment design instruments offer a realm to generate, visualise and analyse urban form. Our research hypothesises that engaging stakeholders using a virtual reality (VR) design platform can reduce the gap between design intent and design outcomes leading to a more favourable design process. The recent development

of VR instruments and accepting them in the design process has brought about a significant shift from utopian design approaches to a more systematic design approach. These instruments offer a ubiquitous interaction platform to generate and visualise iterative design ideas. In our previous work, we argue that a computational design process could accommodate urban complex parameters in a virtual platform for design discussion [1]. As a case study, we have considered a low-density suburb of Wellington, called Karori. The centre of Karori is earmarked for redevelopment to achieve a middensity and mixed-used urban character.

\section{Research Methodology}

The research methodology comprises three steps. It starts by identifying the design problem, then the development of a design instrument and ends with participatory design engagement within VR (Figure 1). From the consultation report of the Wellington City Council (WCC) on Medium Density Residential Area (MDRA),
Karori's community is looking for a thriving centre surrounded by a shopping mall, and its existing Community Hall and Library (Wellington.govt.nz, 2018). Therefore, in the first step, we have identified the contextual design criteria for Karori. In the second step we have developed a parametric integrated model. Finally, the participatory design engagement takes place with urban designers and the local community. The parameters we have chosen arrived from data that we distilled from the MDRA, namely land-division, construction cost, anticipated revenue and area of functional arrangement.

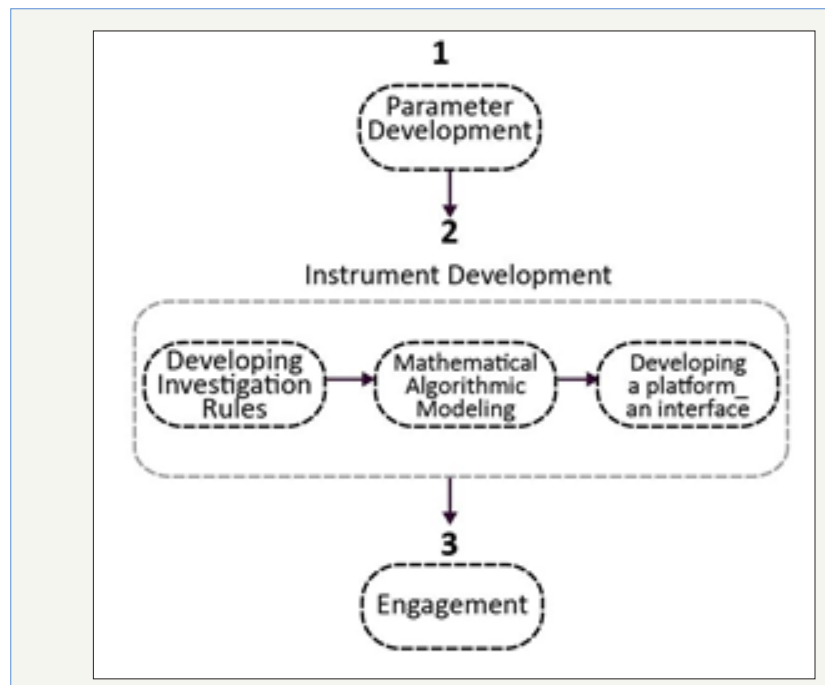

Figure 1: Research framework with its three steps. 


\section{Instrument development}

The design instrument is developed based on the relationship of urban parameters. The method of instrument development is Object Oriented Parametric Modelling (OOPM). The script to generate the urban model and the parametric dependencies is written in a visual programming language, 'Grasshopper3D'. The model is linked to data of the site using GIS or Open Street Map (OSM) generated vector maps. At last, we generate an online interface that allows the visualisation and interactions of different urban scenarios (Figure 2). The generation of scenarios of various urban forms depends on the relationship of the given parameters that compute the urban massing model. In parallel, we have developed an immersive VR version of the instrument that is based on 'Unity Technologies' and the modelling suite described by Innes et al. [2] (Figure 3). Both instruments have been employed in participatory urban design processes with the community of Karori to allow for a bottom-up engagement. The outcomes have shown that participants are successful in expressing their desires and making in design decisions of their own neighbourhood.

Figure 2: Screenshot of the online design instrument with sliders to manipulate the various parameters, which change the displayed urban massing models.
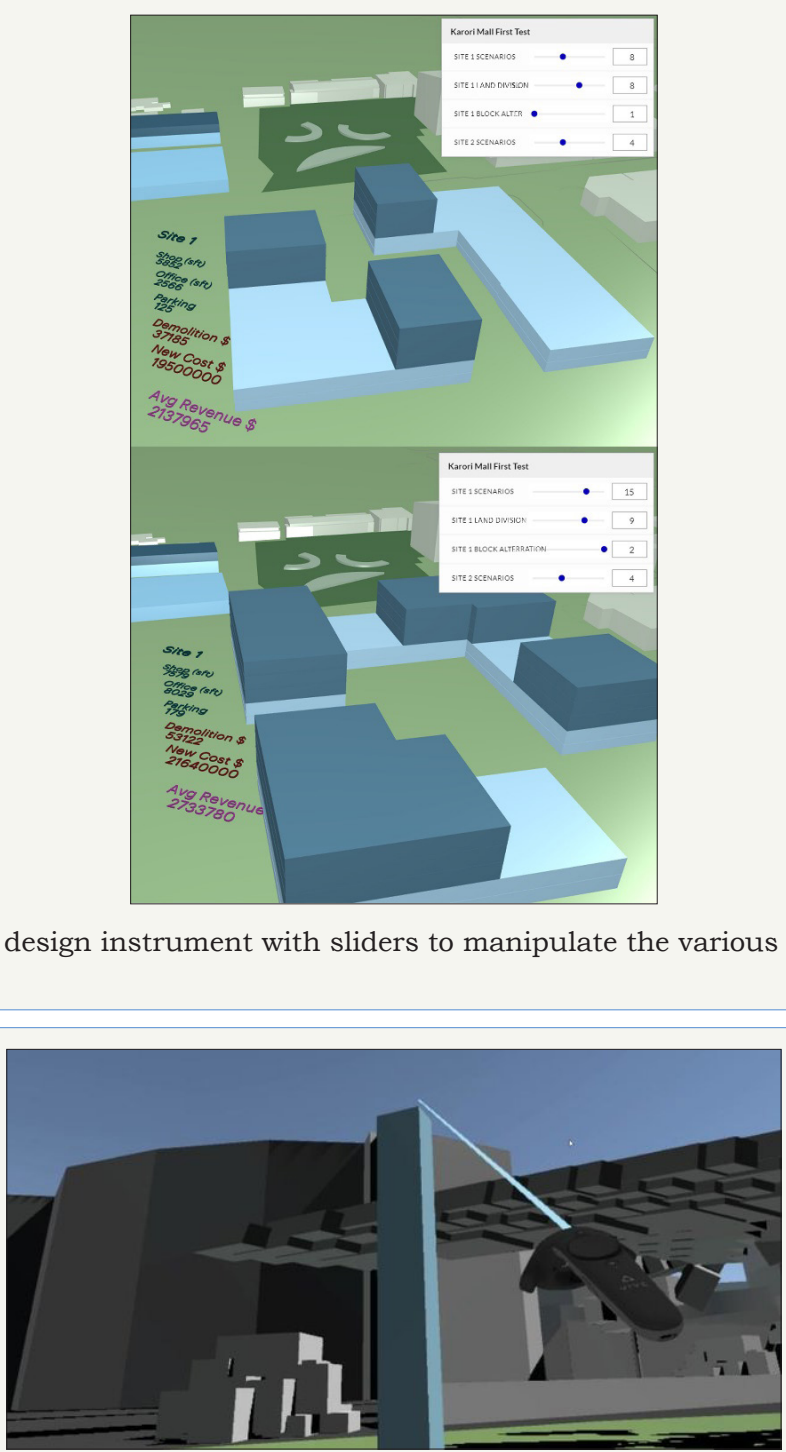

Figure 3: Screenshot of immersive VR of our urban design instrument.

\section{Discussion and Conclusion}

Recent developments in VR allow for novel engagement in participatory urban design. Despite that any design approach cannot include and address all items that are relevant or needed during a design process, VR design instruments offer additionality via their real-time generation and visualisation possibilities that are unmatched in conventional realms. Our here presented instruments for urban design decision-making processes illustrate how VR enable participatory design engagements to reduce the gap between top-down and bottom-up urban design processes.

\section{References}

1. Chowdhury S, Schnabel MA (2018) An algorithmic methodology to predict urban form. In: Fukuda T, Huang W, Janssen P, Crolla K, Alhadidi S (eds.), Learning, Prototyping and Adapting, Learning, $23^{\text {rd }}$ International Conference of the Association for Computer-Aided Architectural Design Research in Asia (CAADRIA), Tsinghua University, Beijing, China, Volume 2, pp. 401-410. 
2. Innes D, Moleta T, Schnabel MA (2017) Virtual inhabitation and creation: a comparative study of interactive 1:1 modelling as a design method. In: Proceedings of 2017 National Conference on Digital Architecture Technologies in Architectural Education and Digital Architecture Design Association (DADA), International Conference on Digital Architecture, Beijing: China Architecture \& Building Press, pp. 402-408.

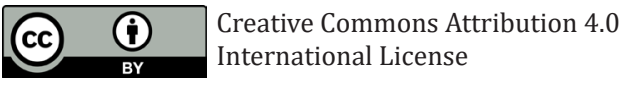

For possible submissions Click Here

\begin{tabular}{|l} 
BCETIvancements in Civil Engineering \& Technology \\
Benefits of Publishing with us
\end{tabular}

